Takashi Nomiyama • Yasushi Tanaka • Lianshan Piao

Keiko Nagasaka • Ken Sakai · Takeshi Ogihara

Kunihiro Nakajima • Hirotaka Watada - Ryuzo Kawamori

\title{
The polymorphism of manganese superoxide dismutase is associated with diabetic nephropathy in Japanese type 2 diabetic patients
}

Received: August 5, 2002 / Accepted: December 3, 2002

\begin{abstract}
We evaluated the relationship of an alanine or valine polymorphism at amino acid sequence 16 [Val(16)Ala] of manganese superoxide dismutase (MnSOD) with diabetes and diabetic nephropathy in Japanese type 2 diabetic patients. Val(16)Ala genotyping of Mn-SOD was done by polymerase chain reaction-restriction fragment length polymorphism with a restriction enzyme (Bsaw I) in 478 Japanese type 2 diabetic patients and 261 nondiabetic Japanese healthy subjects. The genotype distribution of diabetic and nondiabetic subjects was then compared, and the association of genotype with diabetic nephropathy was evaluated in the diabetic patients. The allele frequency and genotype of the diabetic patients were not different from those of the healthy nondiabetic subjects. The VV type showed a significantly higher frequency in the diabetic patients with nephropathy than did the AA or VA type [VV type: normoalbuminuria $70.8 \%$, microalbuminuria $84.8 \%$ $(\mathrm{P}=0.0057)$, macroalbuminuria $84.1 \%(\mathrm{P}=0.0128)]$. Furthermore, logistic regression analysis showed that this polymorphism is associated with diabetic nephropathy independently (odds ratio $=0.461925, \mathrm{P}=0.03$ ). Accordingly, the $\mathrm{Val}(16)$ Ala polymorphism of Mn-SOD may be unrelated to the etiology of type 2 diabetes, but it seems to be associated with diabetic nephropathy in Japanese type 2 diabetic patients.
\end{abstract}

Key words Mn-SOD · Polymorphism · ROS · Mitochondria $\cdot$ Diabetic nephropathy

T. Nomiyama $\cdot$ Y. Tanaka $(\bowtie) \cdot K$. Nagasaka $\cdot$ K. Sakai

T. Ogihara $\cdot$ K. Nakajima $\cdot$ H. Watada $\cdot$ R. Kawamori

Department of Medicine, Metabolism and Endocrinology, School of Medicine, Juntendo University, 2-1-1 Hongo, Bunkyo-ku, Tokyo

113-8421, Japan

Tel. +81-3-5802-1579; Fax +81-3-3813-5996

e-mail: y-tanaka@med.juntendo.ac.jp

L. Piao

Department of Endocrinology, The Affiliated Hospital of Yanbian

University College of Medicine, Yanji, Jilin, China

\section{Introduction}

The mitochondria are the major source of intracellular reactive oxygen species (ROS) because large amounts of superoxide anions are formed as a by-product of the oxidative phosphorylation reactions that occur in the mitochondrial respiratory chain (Guidot et al. 1993). Usually, such ROS are immediately scavenged by mitochondrial manganese superoxide dismutase (Mn-SOD; Weisiger and Fridovich 1973), which may be an essential enzyme for cell survival because disruption of the Mn-SOD gene causes neonatal death in mice (Li et al. 1995). The gene is not coded in the mitochondrial DNA but in the nuclear DNA, and the enzyme translocates to the mitochondria after translation of the protein in the cytosol. Recently, a valine (GTT) or alanine (GCT) polymorphism of human Mn-SOD was found at amino acid position 16 [Val(16)Ala], alternatively described as Val(-9)Ala of the signal peptides in the previous report, which plays a key role in targeting the enzyme to the mitochondria (Shimoda-Matsubayashi et al. 1996). The valine-to-alanine substitution induces a conformational change of the targeting sequence from a $\beta$-sheet to an $\alpha$ helix, and an in vitro study has suggested that the enzyme content in the mitochondrial fraction is higher when the signal peptide has alanine as the position 16 amino acid than when it has valine (Hiroi et al. 1999). Accordingly, a valineto-alanine substitution may increase the targeting efficiency by a conformational change of the targeting sequence, consequently leading to an increase in mitochondrial ROS scavenging.

Hyperglycemia may increase ROS production (West 2000), which may be closely associated with diabetic complications. In particular, ROS produced by mitochondria in a high-glucose condition may regulate various factors relating to diabetic complications (Nishikawa et al. 2000). Among the various diabetic complications, nephropathy is particularly thought to be linked to multiple genetic factors such as the angiotensin converting enzyme insertion or deletion [ACE (I/D)] genotype (Ohno et al. 1996), but there has been no previous report of an association between 
nephropathy and ROS-related genotypes. Accordingly, we analyzed the $\mathrm{Val}(16)$ Ala polymorphism of Mn-SOD in type 2 diabetic patients and healthy nondiabetic subjects. We evaluated the association of genotype with diabetic nephropathy, while simultaneously analyzing the ACE polymorphism, an intron $16 \mathrm{I} / \mathrm{D}$, to avoid its confounding influence.

\section{Patients and methods}

A total of 478 Japanese type 2 diabetic patients ( 315 men and 163 women aged $59.8 \pm 0.5$ years, mean \pm SEM), who were outpatients of Juntendo University Hospital (Tokyo, Japan), and a total of 261 nondiabetic healthy Japanese subjects $(156$ men and 101 women aged $48.1 \pm 0.5$ years, HbA1c $<5.0 \%)$ were studied. All subjects gave written informed consent before enrollment in the study, which was approved by the Ethics Committee of Juntendo University. Hypertension was defined as a systolic blood pressure $>140 \mathrm{mmHg}$ and/or a diastolic blood pressure $>90 \mathrm{mmHg}$ or the use of oral antihypertensive therapy. The stage of diabetic nephropathy was determined from the average of at least two measurements of urinary albumine creatinine ratio (ACR), and the subjects were classified into the following three groups: a normoalbuminuria group $[\mathrm{DN}(-)$ group, ACR $<30 \mathrm{mg} / \mathrm{g}$ creatinine (Cre)], a microalbuminuria group (DN1 group, ACR $\geqq 30 \mathrm{mg} / \mathrm{g}$ Cre and $<300 \mathrm{mg}$ / g Cre), and a macroalubuminuria group (DN2 group, ACR $\geqq 300 \mathrm{mg} / \mathrm{g}$ Cre).

Genomic DNA was extracted from peripheral blood cells using a DNA extraction kit (QIAamp DNA Blood Kit, QIAGEN, Tokyo, Japan). Genotyping of Val(16)Ala of $\mathrm{Mn}-\mathrm{SOD}$ was done by polymerase chain reaction (PCR) restriction fragment length polymorphism analysis. In brief, genomic DNA was amplified using a forward primer (5'-GCTGTGCTTTCTCGTCTTCAG-3') and a reverse primer (5'-TGGTACTTCTCCTCGGTGACG-3'). The PCR involved 38 cycles of $94^{\circ} \mathrm{C}$ for $30 \mathrm{~s}, 60^{\circ} \mathrm{C}$ for $30 \mathrm{~s}$ and $72^{\circ} \mathrm{C}$ for $30 \mathrm{~s}$. Then the PCR products were digested overnight at $60^{\circ} \mathrm{C}$ with $B s a$ w I (New England Biolabs, Beverly, MA, USA), electrophoresed on $2.5 \%$ agarose gel, and stained with ethidium bromide. The ACE (I/D) polymorphism was also examined by PCR analysis, as described previously (Ohno et al. 1996). In brief, genomic DNA was amplified using a forward primer (5'-CTGGAG ACCACTCCCATCCTTTCT-3') and a reverse primer (5' GATGTGGCCATC ACATTCGTCAGAT-3'), after which the PCR products were electrophoresed on $2 \%$ agarose gel and stained with ethidium bromide. Insertion and deletion of the allele was detected as a band of $490 \mathrm{bp}$ and $190 \mathrm{bp}$, respectively.

Results are reported as the mean \pm SEM. The statistical significance of differences in mean values was analyzed by the student's $t$-test and the $\chi^{2}$ test, respectively. To assess the relationship between diabetic nephropathy and the $\mathrm{Mn}$ SOD genotype or other variables, we performed logistic regression analysis.

\section{Results}

Age and $\mathrm{HbA}_{1 \mathrm{C}}$ were significantly higher in the diabetic patients than in the healthy subjects $(P<0.0001$; age, 59.8 \pm 0.5 vs $48.1 \pm 0.5$ years; HbA1c: $7.3 \pm 0.1$ vs $4.5 \pm 0.02$ ), whereas both the allele frequency and the genotype distribution were not significantly different between the two groups \{allele frequency [V/A], $0.879 / 0.122$ vs $0.856 / 0.143$; genotype [VV/VA/AA (\%)], 76.2/23.2/0.6 vs 72.8/25.7/1.5; diabetic patients vs healthy subjects\}.

As shown in Table 1, the frequency of genotype in each group was consistent with the Hardy-Weinberg equilibrium. Clinical characteristics of the diabetic patients stratified by the stage of diabetic nephropathy are also shown in Table 1. Duration of diabetes and HbA1c were higher in the DN1 group compared with the $\mathrm{DN}(-)$ group (duration of diabetes, $P=0.0047$; HbA1c, $P=0.0274$ ). Plasma high-density lipoprotein cholesterol (HDL-C) was lower in the DN1 group compared with the $\mathrm{DN}(-)$ group $(P<0.0001)$. In the DN2 group, body mass index (BMI), duration of diabetes, $\mathrm{HbA} 1 \mathrm{c}$, and plasma total choleskrol (T-CHO) were higher than in the DN $(-)$ group (BMI, $P=$ 0.0084; duration of diabetes, $P=0.0001$; HbA1c, $P=$ 0.0136 ; T-CHO, $P=0.0021$ ), and the onset age of diabetes and HDL-C were lower than in the $\mathrm{DN}(-)$ group (onset age of diabetes, $P=0.03$; HDL-C, $P=0.0419)$. Furthermore, $\mathrm{T}-\mathrm{CHO}$ in the DN2 group was higher than in the DN1 group $(P=0.0048)$. The rate of hypertension was higher in a severe stage of nephropathy $[\mathrm{DN}(-)$ vs $\mathrm{DN} 1$, $P=0.0127 ; \mathrm{DN} 1$ vs DN2, $P=0.0197]$. The frequency of the VV genotype of Mn-SOD was higher in the nephropathy group $(\mathrm{DN}(-)$ vs $\mathrm{DN} 1 ; P=0.0057, \mathrm{DN}(-)$ vs $\mathrm{DN} 2 ; P=$ $0.0128)$. There was no difference of ACE genotype distribution among the groups.

To concentrate on the difference in the Mn-SOD genotype between the presence and absence of nephropathy, we further performed a similar comparison between the subjects without nephropathy [nephropathy $(-), n=291$ ] and the combined subjects of the DN1 and DN2 groups [nephropathy $(+), n=187$ ]. As shown in Table 2 , the frequencies of the Mn-SOD genotype in these groups were consistent with the Hardy-Weinberg equilibrium. In the nephropathy (+) group, BMI, duration of diabetes, $\mathrm{HbA} 1 \mathrm{c}$, and T-CHO were significantly higher and the onset age of diabetes and HDL-C were significantly lower than for those in the nephropathy $(-)$ group. Although the ACE genotype was not different between the two groups, the VV genotype of MnSOD was significantly higher in the nephropathy (+) group than that in the nephropathy (-) group $(84.5 \%$ vs $70.8 \%$, $P=0.0006$ ).

Logistic regression analysis was done to assess the relationship between progression of nephropathy from normoalbuminuria to macroalbuminuria and the clinical factors or the Mn-SOD genotype. As shown in Table 3, the estimated duration of diabetes, T-CHO, HDL-C, hypertension, and genotype of Mn-SOD were significantly associated with nephropathy. Although the ACE(I/D) genotype was not associated with nephropathy in the present study, 
Table 1. Characteristics of the diabetic patients stratified by stage of nephropathy

\begin{tabular}{llll}
\hline DN stage & $\mathrm{DN}(-)$ & $\mathrm{DN} 1$ & $\mathrm{DN} 2$ \\
$n$ & 291 & 99 & 88 \\
Sex (male/female) & $191 / 100$ & $66 / 33$ & $67 / 21$ \\
Age (years) & $59.3 \pm 0.6$ & $61.0 \pm 1.1$ & $60.2 \pm 1.3$ \\
BMI (kg/m ${ }^{2}$ ) & $22.8 \pm 0.2$ & $23.0 \pm 0.4$ & $24.0 \pm 0.5^{\mathrm{a}}$ \\
Duration of diabetes (years) & $10.6 \pm 0.5$ & $13.6 \pm 1.0^{\mathrm{a}}$ & $14.7 \pm 1.0^{\mathrm{a}}$ \\
Onset age of diabetes (years) & $49.0 \pm 0.7$ & $47.5 \pm 1.3$ & $45 \pm 1.5^{\mathrm{b}}$ \\
HbA1c (\%) & $7.1 \pm 0.1$ & $7.5 \pm 0.6^{\mathrm{b}}$ & $7.5 \pm 0.2^{\mathrm{b}}$ \\
T-CHO (mg/dl) & $192 \pm 2.2$ & $191.2 \pm 4.0$ & $208.2 \pm 5.1^{\mathrm{a}, \mathrm{c}}$ \\
HDL-C (mg/dl) & $55.8 \pm 1.0$ & $52.2 \pm 1.5^{\mathrm{a}}$ & $52.4 \pm 1.7^{\mathrm{b}}$ \\
TG (mg/dl) & $159.4 \pm 10.1$ & $165.1 \pm 9.9$ & $220.6 \pm 35.5$ \\
HT [(-)/(+) (\%)] & $199(68.4) / 92(31.6)$ & $54(54.5) / 45(45.5)^{\mathrm{d}}$ & $33(37.5) / 55(62.5)^{\mathrm{e}, \mathrm{f}}$ \\
Mn-SOD genotype [AA,VA/VV (\%)] & $85(29.3) / 206(70.8)$ & $15(15.1) / 84(84.8)^{\mathrm{e}}$ & $14(15.9) / 74(84.1)^{\mathrm{d}}$ \\
Hardy-Weinberg equilibrium $(P$ value) & 0.06073 & 0.88878 & 0.85147 \\
ACE genotype [II/ID, DD $(\%)]$ & $118(40.5) / 173(59.5)$ & $45(45.5) / 54(54.5)$ & $34(38.6) / 54(61.4)$ \\
\hline
\end{tabular}

Data are means \pm SEM

$\mathrm{DN}(-)$, normoalbuminuria; DN1, microalbuminuria; DN2, macroalbuminuria; BMI, body mass index; HbA1c, hemoglobin A1c; T-CHO, plasma total cholesterol; HDL-C, plasma high-density lipoprotein-cholesterol; TG, plasma triglyceride; HT, hypertension; Mn-SOD, manganese Superoxide dismutase; ACE, angiotensin converting enzyme

${ }^{a} P<0.01$ vs $\mathrm{DN}(-)$ by student's $t$-test

${ }^{\mathrm{b}} P<0.05$ vs DN $(-)$ by student's $t$-test

${ }^{\mathrm{c}} P<0.01$ vs DN1 by student's $t$-test

${ }^{\mathrm{d}} P<0.05$ vs $\mathrm{DN}(-)$ by $\chi^{2}$ test

${ }^{\mathrm{e}} P<0.01$ vs $\mathrm{DN}(-)$ by $\chi^{2}$ test

${ }^{\mathrm{f}} P<0.05$ vs DN1 by $\chi^{2}$ test

Table 2. Comparison of characteristics and genotypes between the groups with and without nephropathy

\begin{tabular}{lll}
\hline DN stage & Nephropathy(-) & Nephropathy $(+)$ \\
$n$ & 291 & 187 \\
Sex (male/female) & $191 / 100$ & $124 / 63$ \\
Age (years) & $59.3 \pm 0.6$ & $60.6 \pm 0.8$ \\
BMI (kg/m ${ }^{2}$ ) & $22.8 \pm 0.2$ & $23.5 \pm 0.3^{\mathrm{a}}$ \\
Duration of diabetes (years) & $10.6 \pm 0.5$ & $14.1 \pm 0.7^{\mathrm{b}}$ \\
Onset age of diabetes (years) & $49.0 \pm 0.7$ & $47.0 \pm 1.0^{\mathrm{a}}$ \\
HbA1c $(\%)$ & $7.1 \pm 0.1$ & $7.5 \pm 0.1^{\mathrm{b}}$ \\
T-CHO (mg/dl) & $192 \pm 2.2$ & $199.2 \pm 3.3^{\mathrm{b}}$ \\
HDL-C (mg/dl) & $55.8 \pm 1.0$ & $52.3 \pm 1.1^{\mathrm{b}}$ \\
TG (mg/dl) & $159.4 \pm 10.1$ & $191.2 \pm 17.6$ \\
HT [(-)/(+) $(\%)]$ & $199(68.4) / 92(31.6)$ & $87(46.5) / 100(43.5)^{\mathrm{c}}$ \\
Mn-SOD genotype [AA,VA/VV (\%)] & $85(29.3) / 206(70.8)$ & $29(15.5) / 158(84.5)^{\mathrm{d}}$ \\
Hardy-Weinberg equilibrium of Mn-SOD & & \\
$\quad$ genotype $(P$ value) & 0.06073 & 0.785483 \\
ACE genotype [II/ID,DD (\%)] & $118(40.5) / 173(59.5)$ & $79(42.2) / 108(57.8)$ \\
\hline
\end{tabular}

Data are means \pm SEM

For abbreviations, see Table 1

${ }^{\text {a }} P<0.05$ vs nephropathy $(-)$ by student's $t$-test

${ }^{\mathrm{b}} P<0.01$ vs nephropathy ( - ) by student's $t$-test

${ }^{\mathrm{c}} P<0.01$ and ${ }^{\mathrm{d}} P<0.001$ vs nephropathy $(-)$ by $\chi^{2}$ test

Table 3. Logistic regression analysis of factors related to nephropathy

\begin{tabular}{|c|c|c|c|c|}
\hline Variable & Partial regression coefficient & Odds ratio & $95 \% \mathrm{CI}$ & $P$ \\
\hline Age (years) & 0.000919637 & 1.00092 & $0.97-1.03$ & 0.95 \\
\hline BMI $\left(\mathrm{kg} / \mathrm{m}^{2}\right)$ & 0.0711933 & 1.07379 & $0.99-1.15$ & 0.07 \\
\hline Duration of diabetes (years) & 0.0497678 & 1.05103 & $1.02-1.09$ & $<0.01$ \\
\hline $\mathrm{HbA} 1 \mathrm{c}(\%)$ & 0.170691 & 1.18612 & $0.98-1.44$ & 0.09 \\
\hline T-CHO (mg/dl) & 0.010453 & 1.01051 & $1.00-1.02$ & 0.02 \\
\hline HDL-C (mg/dl) & -0.00745423 & 0.992573 & $0.97-1.01$ & 0.43 \\
\hline $\mathrm{TG}(\mathrm{mg} / \mathrm{dl})$ & -0.00025131 & 0.999749 & $0.99-1.00$ & 0.74 \\
\hline Hypertension $[0:(-), 1:(+)]$ & 1.18709 & 3.27754 & $1.90-5.65$ & $<0.01$ \\
\hline ACE [0:(II), 1:(ID, D/D)] & 0.207697 & 1.23084 & $0.70-2.15$ & 0.46 \\
\hline Mn-SOD [0:(VV), 1:(VA, AA)] & -0.772353 & 0.461925 & $0.23-0.93$ & 0.03 \\
\hline
\end{tabular}

CI, Confidence interval. For other abbreviations, see Table 1 
the VA or AA genotype of Mn-SOD was associated with nephropathy $(P=0.03)$.

\section{Discussion}

In the present study, we analyzed the $\mathrm{Val}(16)$ Ala polymorphism of Mn-SOD in Japanese type 2 diabetic patients and healthy nondiabetic subjects, and then evaluated the association of this polymorphism with nephropathy in the diabetic patients. Although the overall allele frequency did not differ between the diabetic patients and the nondiabetic subjects, the AA or VA genotype was significantly rarer in the diabetic patients with nephropathy than in those without nephropathy. Because microalbuminuria is clinically not independent of normoalbuminuria or macroalbuminuria and can be reversible to such stages by short-term influence of blood pressure or glycemic control, we omitted this stage from regression analysis to strictly evaluate the effect of the Mn-SOD genotype in nephropathy. The regression analysis showed that the Mn-SOD genotype but not the ACE genotype significantly correlated to nephropathy. Furthermore, although we evaluated the effect of the combinational genotype of ACE and Mn-SOD, neither an additive nor a synergistic effect of such a combination on nephropathy was observed (data not shown). Thus, the association of the Mn-SOD genotype with nephropathy may be independent of the ACE genotype.

High glucose levels can induce an excess of ROS through the nonenzymatic formation of advanced glycation endproducts (AGE), glucose autooxidation, and increased activity of the polyol pathway. However, Nishikawa et al. (2000) previously reported that an increase of ROS in cultured vascular endothelial cells cultured under high-glucose conditions was prevented by the inhibition of electron transport chain complex II, by uncoupling oxidative phosphorylation, by uncoupling protein-1, and by Mn-SOD. These findings suggest that the increase of ROS in hyperglycemic states may be mainly related to mitochondrial dysfunction. Accordingly, Mn-SOD, which is the pivotal ROS scavenging enzyme in mitochondria, seems to play a key role in preventing the ROS-related complications of diabetes.

Very recently, Chistyakov et al. (2001) reported that the Mn-SOD allele frequency did not differ between Russian type 1 diabetic and nondiabetic subjects and that the $\mathrm{V}$ allele was significantly associated with neuropathy, but not with retinopathy or nephropathy. However, the V allele frequency in our Japanese healthy nondiabetic subjects was remarkably higher than in the control Russian subjects $(85.6 \%$ vs $34.1 \%)$ and the type of diabetes studied was also different. Although we cannot understand why the frequencies of Mn-SOD variants (AA + VA) were lower in the nephropathy group than were those in the nonnephropathy group, decreased AA and VA frequencies were also reported in other diseases such as schizophrenia (Hori et al. 2000), cardiomyopathy (Hiroi et al. 1999), or asbestosassociated pulmonary disorders (Hirvonen et al. 2002). A possible interpretation is that the A allele may be an active type for scavenging activity in mitochondria and thus it may prevent ROS-related diseases. However, we do not have further information concerning other possibilities. Further large-scale cross-sectional and prospective studies are required to clarify the pathological association of the MnSOD Val(16)Ala polymorphism with the development and progression of diabetic complications.

Another site of polymorphism of Mn-SOD, isoleucine (Ile) or threonine (Thr) at amino acid 58 (Ile58Thr), was reported previously (Ho and Crapo 1998). Mn-SOD with 58Thr, as well as Mn-SOD with $16 \mathrm{Val}$, may be a weaker antioxidant than the native enzyme. Such considerations indicate that it may also be valuable to investigate the significance of the Ile58Thr polymorphism in diabetic subjects.

In conclusion, the Val(16)Ala polymorphism of MnSOD may be unrelated to the etiology of type 2 diabetes. However, this polymorphism seems to be associated with nephropathy in type 2 Japanese diabetic patients.

\section{References}

Chistyakov DA, Savost'anov KV, Zotova EV, Nosikov VV (2001) Polymorphisms in the $M n-S O D$ and $E C-S O D$ genes and their relationship to diabetic neuropathy in type 1 diabetes mellitus. BMC Med Genet 2:4

Guidot DM, McCord JM, Wright RM, Repine JE (1993) Absence of electron transport (Rho 0 state) restores growth of a manganesesuperoxide dismutase-deficient Saccharomyces cerevisiae in hyperoxia. Evidence for electron transport as a major source of superoxide generation in vivo. J Biol Chem 268:26699-26703

Hiroi S, Harada H, Nishi H, Satoh M, Nagai R, Kimura A (1999) Polymorphisms in the SOD2 and $H L A-D R B 1$ genes are associated with nonfamilial idiopathic dilated cardiomyopathy in Japanese. Biochem Biophys Res Commun 261:332-339

Hirvonen A, Tuimala J, Ollikainen T, Linnainmaa K, Kinnula V (2002) Manganese superoxide dismutase genotypes and asbestos-associated pulmonary disorders. Cancer Lett 178:71-74

Ho YS, Crapo JD (1998) Isolation and characterization of complementary DNAs encoding human manganese-containing superoxide dismutase. FEBS Lett 229:256-260

Hori H, Ohmori O, Shinkai T, Kojima H, Okano C, Suzuki T, Nakamura J (2000) Manganese superoxide dismutase gene polymorphism and schizophrenia: relation to tardive dyskinesia. Neuropsychopharmacology 23:170-177

Li Y, Huang TT, Carlson EJ, Melov S, Ursell PC, Olson JL, Noble LJ, Yoshimura MP, Berger C, Chan PH, Wallace DC, Epstein CJ (1995) Dilated cardiomyopathy and neonatal lethality in mutant mice lacking manganese superoxide dismutase. Nat Genet 11:376-381

Nishikawa T, Edelstein D, Du XL, Yamagishi S, Matsumura T, Kaneda Y, Yorek MA, Beebe D, Oates PJ, Hammes P, Giardino I, Brownlee M (2000) Normalizing mitochondrial superoxide production blocks three pathways of hyperglycaemic damage. Nature 404:787-790

Ohno T, Kawazu S, Tomono S (1996) Association analyses of the polymorphisms of angiotensin-converting enzyme and angiotensinogen genes with diabetic nephropathy in Japanese non-insulin-dependent diabetics. Metabolism 45:218-222

Shimoda-Matsubayashi S, Matsumine H, Kobayashi T, NakagawaHattori Y, Shimizu Y, Mizuno Y (1996) Structural dimorphism in the mitochondrial targeting sequence in the human manganese superoxide dismutase gene. A predictive evidence for conformational change to influence mitochondrial transport and a study of allelic association in Parkinson's disease. Biochem Biophys Res Commun 226:561-565

Weisiger RA, Fridovich I (1973) Superoxide dismutase. Organelle specificity. J Biol Chem 248:3582-3592

West IC (2000) Radicals and oxidative stress in diabetes. Diabet Med 17:171-180 